\title{
Construction and Test of Thermostats and Twirlers for Molecular Rotations
}

\author{
Siegfried Hess \\ Institut für Theoretische Physik, Technische Universität Berlin, \\ PN 7-1, Hardenbergstr. 36, D-10623 Berlin, Germany \\ Reprint requests to S. H.; S.Hess@ physik.tu-berlin.de \\ Z. Naturforsch. 58a, 377 - 391 (2003); received May 28, 2003
}

The equations of motion are coupled with a dynamical variable, referred to as twirler, which randomizes the angular momentum. The equations are time-reversal invariant, just as those for the standard Gaussian, Nosé-Hoover and configurational thermostats. The derivation of the basic equations is outlined. Test calculations are performed for the two-dimensional isotropic harmonic oscillator and for a nonlinear elastic dumbbell, used as a simple model to study properties of polymer molecules. Graphs of characteristic quantities and orbits, some of which are rather intriguing, are displayed. As applications, the rotational diffusion and the influence of a shear flow on the angular velocity and the deformation of the model polymer are analyzed.

Key words: Molecular Rotations; Dumbbell; Thermostats; Diffusion; Shear Flow.

\section{Introduction}

Time-reversible thermostats such as the Gaussian isokinetic thermostat and those due to Nosé and Hoover [1-5] which imply a behavior consistent with a canonical distribution in equilibrium, as well as generalisations thereof $[6,7]$, including the configurational thermostat [8], have found widespread applications in equilibrium and non-equilibrium molecular dynamics computer simulations $[1-5,9]$ for systems containing many (typically $10^{2}$ to $10^{6}$ ) particles. When applied to systems of few particles in two or three dimensions it is desireable to have a "stirring", "spinning" or "twirling" (rotational rocking) mechanism which affects the directions of the velocities and not just their magnitudes as all standard thermostats do. This point is particularly evident for the extendable dumbbell subjected to a simple shear flow which has recently been used as a model [10] to study the shear induced rotation and deformation of polymer molecules [11]. A term is needed in the equation of motion analogous to the Lorentz force involving a fluctuating magnetic field which obeys an additional differential equation.

In this article, firstly some general remarks are made on the derivation of equations governing thermostats and on the construction of a dynamic stirring mechanism referred to as "twirler". Then a special choice for a twirler or "directional thermostat" is introduced and some applications for a two-dimensional isotropic harmonic oscillator and for the dumbbel problem are presented. More specifically, it is tested how the desired properties of the twirler, like randomization of the angular momentum and values of time averages expected for a canonical distribution, depend on the size of the coupling coefficient in the additional dynamic equation. Combinations of the twirler with Gaussian, NoséHoover, force-momentum and configurational thermostats are considered. Examples of orbits are displayed. These are particularly intriguing for the adiabatic twirler applied to the harmonic oscillator, some graphs are presented in the appendix. For the dumbbell, it is demonstrated that the twirler, combined with the Gaussian thermostat, leads to a rotational diffusive behavior when an average over the initial conditions is performed. Furthermore, it is shown that it is possible to treat the influence of a shear flow on the rotational behavior and on the deformation of a model polymer molecule by the present method.

The approach introduced here is complementary to the kinetic theory methods used to study the coupling between rotational and translational motions in gases [12-15] liquids [15] and colloidal dispersions [16]. In Kinetic Theory, one starts from equations which have the irreversibility of the processes studied already build in, whereas here, as emphasised before, time-reversible equations are employed. Similarly, a Langevin equa- 
tion with fluctacting forces would also randomize the angular momentum. There, however, the irreversibility of the equation is build in due to the use of a friction coefficient.

\section{On the Construction of Thermostats and "Twirlers"}

\subsection{General Remarks}

For simplicity one particle in $D$ spatial dimensions, with $D=2$ or $D=3$, is considered. The position and momentum variables are $\boldsymbol{r}$ and $\boldsymbol{p}$. The force $\boldsymbol{F}=\boldsymbol{F}(\boldsymbol{r})$ acting on the particle is assumed to be derived from an interaction potential $\Phi$ which provides characteristic length and energy scales. With the help of them and the mass $m$ of the particle, all physical quantities of interest, including the time $t$ and the temperature $T$, can be given as multiples of reference values. For the dimensionless reduced variables the same symbols are used as for the corresponding physical variables. Then, the equation of motion for the constraint free situation is equivalent to $\dot{\boldsymbol{r}}=\boldsymbol{p}, \dot{\boldsymbol{p}}=\boldsymbol{F}$. Terms associated with a flow velocity will be added later. Thermostats and the twirler impose constraints on the dynamics.

The configurational thermostat is a special case of a "r-thermostat" which modifies the equation for $\dot{\boldsymbol{r}}$ viz.:

$$
\dot{\boldsymbol{r}}=\boldsymbol{p}-\phi(\boldsymbol{r}, \boldsymbol{p}) \kappa \boldsymbol{r}
$$

Here the attention is focused on modifications of the momentum equation. To include the action of a " $p$ thermostat" and a twirler, the equations of motion are replaced by

$$
\dot{\boldsymbol{p}}=\boldsymbol{F}-\varphi(\boldsymbol{r}, \boldsymbol{p}) \alpha \boldsymbol{p}+\psi(\boldsymbol{r}, \boldsymbol{p}) \boldsymbol{W} \times \boldsymbol{p} .
$$

The first additional term in the momentum equation which is proportional to $\boldsymbol{p}$ comprises the Gaussian, the Nosé-Hoover and the "p4" thermostats as well as the "force-momentum" thermostat to be introcuced later as special cases corresponding to specific choices for the scalar quantity $\varphi$. Equations for the scalar dynamical variables $\kappa$ and $\alpha$ are stated later. The additional term involving the pseudo-vector $\boldsymbol{W}$ does not affect the magnidute of the momentum but it provides directional changes corresponding the desired stirring motion. The scalar $\psi$ is specified later. Before equations of change for the additional variables $\alpha$ and $\mathbf{W}$ are stated, it is noticed that time reversal invariance of the equation of motion requires that both $\varphi \alpha$ and $\psi \boldsymbol{W}$ change sign under time reversal. The dynamic variables $\kappa, \alpha$ and $\boldsymbol{W}$ are determined by arguments similar to those put forward earlier [4, 6]. The distribution function $f_{\text {eq }}(\boldsymbol{r}, \boldsymbol{p}, \kappa, \alpha, \boldsymbol{W})$, in thermal equilibrium, is assumed to be proportional to

$$
\exp (-\beta H) \exp \left(-\kappa^{2} / 2\right) \exp \left(-\alpha^{2} / 2\right) \exp (-\boldsymbol{W} \cdot \boldsymbol{W} / 2),
$$

with $\beta=1 / T$ and the Hamiltonian $H=p^{2} / 2+\Phi$. The requirement that the equations of motion conserve the extended canonical distribution $f_{\text {eq }}$ implies

$$
\begin{gathered}
\frac{\partial}{\partial \boldsymbol{r}} \cdot\left(\dot{\boldsymbol{r}} f_{\mathrm{eq}}\right)+\frac{\partial}{\partial \boldsymbol{p}} \cdot\left(\dot{\boldsymbol{p}} f_{\mathrm{eq}}\right)+\frac{\partial}{\partial \kappa}\left(\dot{\boldsymbol{\kappa}} f_{\mathrm{eq}}\right) \\
+\frac{\partial}{\partial \alpha}\left(\dot{\alpha} f_{\mathrm{eq}}\right)+\frac{\partial}{\partial \boldsymbol{W}} \cdot\left(\dot{\boldsymbol{W}} f_{\mathrm{eq}}\right)=0
\end{gathered}
$$

This condition, together with (1), (2) and the assumption that $\dot{\kappa}, \dot{\alpha}$ and $\dot{\boldsymbol{W}}$ be independent of $\kappa, \alpha$ and $\boldsymbol{W}$, leads to

$$
\begin{aligned}
\frac{\mathrm{d} \kappa}{\mathrm{d} t} & =-\beta \phi \boldsymbol{F} \cdot \boldsymbol{r}-\left(D \phi+\boldsymbol{r} \cdot \frac{\partial}{\partial \boldsymbol{r}} \phi\right), \\
\frac{\mathrm{d} \alpha}{\mathrm{d} t} & =\beta \varphi p^{2}-\left(D \varphi+\boldsymbol{p} \cdot \frac{\partial \varphi}{\partial \boldsymbol{p}}\right), \\
\frac{\mathrm{d} \boldsymbol{W}}{\mathrm{d} t} & =\boldsymbol{p} \times \frac{\partial}{\partial \boldsymbol{p}} \psi .
\end{aligned}
$$

The coupled equations $(1,2)$ and $(4-6)$ are invariant under time reversal. The relations for $\dot{\kappa}$ and $\dot{\alpha}$ are equivalent to equations presented earlier $[4,6]$. The expression for $\dot{\boldsymbol{W}}$ is new. The special case $\varphi=$ const corresponds to the Nosé-Hoover thermostat. Notice that $\psi=$ const, on the other hand, implies $\boldsymbol{W}=$ const which contradicts the above made assumption that the values of $\boldsymbol{W}$ obey a Gaussian distribution with zero mean. A special choices for $\phi, \varphi$ and $\psi$ are presented and discussed next.

\subsection{Nosé-Hoover, Force-Momentum, and Configurational Thermostats}

The special choice $\varphi=v_{\mathrm{NH}}=$ const with the relaxation frequency coefficient $v_{\mathrm{NH}}$ corresponds to the Nosé-Hoover thermostat. For this case, the pertinent dynamic variable $\alpha$ is denoted by $\alpha_{\mathrm{NH}}$. For large values of $v_{\mathrm{NH}}$ the dynamics approaches that of the (isokinetic) Gaussian thermostat for which one sets $\varphi \alpha=$ $\boldsymbol{F} \cdot \boldsymbol{p} / p^{2}$. This relation guarantees $\boldsymbol{p} \cdot \dot{\boldsymbol{p}}=0$ such that $p^{2}=D T=$ const. The "p4-thermostat" corresponds 
to $\varphi=v_{\mathrm{p} 4} p^{2} / T$ with the relaxation frequency $v_{\mathrm{p} 4}$, the pertinent dynamic variable is denoted by $\alpha_{\mathrm{p} 4}$.

The choice $\varphi=c_{\mathrm{fp}} \boldsymbol{F} \cdot \boldsymbol{p} / T$ with the coupling coefficient $c_{\mathrm{fp}}=$ const and the dynamic variable $\alpha_{\mathrm{fp}}$ is referred to as "force-momentum" (fp) thermostat.

The configurational thermostat corresponds to $\tau_{\mathrm{cf}} r^{-1} \Phi^{\prime}$ with the relaxation time coefficient $\tau_{\mathrm{cf}}$. Notice that $r^{-1} \Phi^{\prime} \boldsymbol{r}=-\boldsymbol{F}$. The extra term in (1) then is proportional to the force $\boldsymbol{F}$. In this case the dynamic variable is denoted by $\kappa_{\mathrm{cf}}$.

\subsection{A Specific Twirler and an Effective Magnetic Field}

The simplest meaningful choice for $\psi$ is the scalar product $\boldsymbol{r} \cdot \boldsymbol{p}$. This implies that $\dot{W}$ becomes proportional to the angular momentum $\boldsymbol{L}=\boldsymbol{r} \times \boldsymbol{p}$. However, the choice $\psi \sim \boldsymbol{F} \cdot \boldsymbol{p}$ seems to be more appropriate since it implies that the twirler affects the dynamics only over distances where the force acts. More specifically, the ansatz

$$
\psi=c_{\mathrm{st}} \boldsymbol{F} \cdot \boldsymbol{p} / T
$$

is made with the (dimensionless) stirring coupling coefficient $c_{\text {st }}$. Then (6) becomes

$$
\frac{\mathrm{d} \boldsymbol{W}}{\mathrm{d} t}=-\left(c_{\mathrm{st}} / T\right) \boldsymbol{F} \times \boldsymbol{p} .
$$

The term involving the $\boldsymbol{W}$ in the equation of motion can be written as

$$
c_{\mathrm{St}} \boldsymbol{B}_{\text {eff }} \times \boldsymbol{p}
$$

with the effective "magnetic field"

$$
\boldsymbol{B}_{\mathrm{eff}}=(\boldsymbol{F} \cdot \boldsymbol{p} \boldsymbol{W}-\boldsymbol{F} \cdot \boldsymbol{W p}) / T .
$$

The coupling coefficient $-c_{\text {st }}$ plays the role of a charge. The second term proportional to $\boldsymbol{p}$ gives no contribution in the equation of motion, however it is needed to have a divergence free effective $B$-field. This effective field is derived from an effective vector potential $\boldsymbol{A}_{\text {eff }}=-\boldsymbol{\Phi} \boldsymbol{W} \times \boldsymbol{p}$ where $\boldsymbol{\Phi}$ is recalled as the interaction potential. Notice that $\boldsymbol{A}_{\mathrm{eff}} \cdot \boldsymbol{p}=0$ where, in the absence of an external flow field, $\boldsymbol{p}$ is just the particle velocity. Thus the action of the stirring cannot be described by a standard Lagrangian or Hamiltionian dynamics of a charged particle in a magnetic field which requires $\boldsymbol{A} \cdot \dot{\boldsymbol{r}} \neq 0$.

\subsection{Equations of Motion}

The equations of motion to be applied in the following are

$$
\dot{\boldsymbol{r}}=\boldsymbol{p}+\tau_{\mathrm{cf}} \kappa_{\mathrm{cf}} \boldsymbol{F},
$$

and, for the stirrer combined with the Gaussian thermostat

$$
\dot{\boldsymbol{p}}=\boldsymbol{F}-\left(\boldsymbol{F} \cdot \boldsymbol{p} / p^{2}\right) \boldsymbol{p}+c_{\mathrm{st}}(\boldsymbol{F} \cdot \boldsymbol{p} / T) \boldsymbol{W} \times \boldsymbol{p} .
$$

For the Nosé-Hoover and force-momentum thermostats, one has

$$
\begin{aligned}
\dot{\boldsymbol{p}}= & \boldsymbol{F}-v_{\mathrm{NH}} \alpha_{\mathrm{NH}} \boldsymbol{p}-c_{\mathrm{fp}} \alpha_{\mathrm{fp}}(\boldsymbol{F} \cdot \boldsymbol{p} / T) \boldsymbol{p} \\
& +c_{\mathrm{st}}(\boldsymbol{F} \cdot \boldsymbol{p} / T) \boldsymbol{W} \times \boldsymbol{p} .
\end{aligned}
$$

The dynamic variable $\boldsymbol{W}$ is govered by (8). The quanties $\alpha$.. obey the equations

$$
\begin{aligned}
& \frac{\mathrm{d} \alpha_{\mathrm{NH}}}{\mathrm{d} t}=v_{\mathrm{NH}}\left(\beta p^{2}-D\right), \\
& \frac{\mathrm{d} \alpha_{\mathrm{fp}}}{\mathrm{d} t}=c_{\mathrm{fp}}(\boldsymbol{F} \cdot \boldsymbol{p} / T)\left(\beta p^{2}-(D+1)\right) .
\end{aligned}
$$

The variable $\kappa_{\mathrm{cf}}$ associated with the configurational thermostat obeys the equation

$$
\frac{\mathrm{d} \kappa_{\mathrm{cf}}}{\mathrm{d} t}=\tau_{\mathrm{cf}}(\boldsymbol{F} \cdot \boldsymbol{F} / T-\Delta \Phi)
$$

The (instantaneous) "configurational" temperature $T_{\text {conf }}$ is defined by $T_{\text {conf }}=\boldsymbol{F} \cdot \boldsymbol{F} / \Delta \boldsymbol{\Phi}[8]$. Notice that one has $\boldsymbol{F} \cdot \boldsymbol{F} / T-\Delta \Phi=\Delta \Phi\left(T_{\mathrm{conf}} / T-1\right)$, i.e. $\kappa_{\mathrm{cf}}$ does not change when the configurational temperature matches the prescribed temperature $T$. Incidendally, the Einstein frequency $\omega_{E}$ is related to the Laplacian $\Delta$ applied on the potential by $D \omega_{E}^{2}=\Delta \Phi$, with the dimension $D=2$ or 3 .

\subsection{Remarks on Coupling Coefficients}

The relaxation frequencies and coupling coefficients such as $v_{\mathrm{NH}}$ and $c_{\mathrm{st}}$ have to be chosen such that physically meaningful results are obtained, mostly intuition as well as trial and error have been employed. An educated guess for an estimate of the stirring coupling coefficient $c_{\mathrm{st}}$ is made next. Firstly, the case is considered where the stirrer is combined with the Gaussian thermostat. Assuming that $\boldsymbol{F}$ is a central force, the time change of the angular momentum $\boldsymbol{L}$ is given by

$$
\dot{\boldsymbol{L}}=-\boldsymbol{F} \cdot \boldsymbol{p} \boldsymbol{L} / p^{2}+\left(c_{\mathrm{st}} / T\right) \boldsymbol{F} \cdot \boldsymbol{p r} \times(\boldsymbol{W} \times \boldsymbol{p}) .
$$


The long time average $\langle\ldots\rangle$ of both sides of this equation vanishes, the same holds true for $\langle\boldsymbol{L}\rangle$ and $\langle\boldsymbol{W}\rangle$. Assuming that the r.h.s. of the above equation is approximately zero one obtains $\boldsymbol{L} \approx\left(c_{\mathrm{st}} / T\right) p^{2} \boldsymbol{r} \times(\boldsymbol{W} \times \boldsymbol{p})$. Squaring both sides of this relation and averaging them leads to $\left\langle\boldsymbol{L}^{2}\right\rangle \approx c_{\mathrm{st}}^{2} T D^{2}(D-1)\left\langle r^{2}\right\rangle\left\langle W^{2}\right\rangle$. For the isokinetic case analyzed here $\left\langle p^{2 \mathrm{n}}\right\rangle=(D T)^{\mathrm{n}}$ was used. Furthermore, the component of $\boldsymbol{W}$ parallalel to $\boldsymbol{p}$ should not be counted, i. e. $\boldsymbol{W} \cdot \boldsymbol{p}=0$, and one has $\left\langle W^{2}\right\rangle=$ $D-1$. Since $\left\langle\boldsymbol{L}^{2}\right\rangle=\theta T$ with the moment of inertia $\theta=(2 / D)\left\langle r^{2}\right\rangle$, these considerations lead to

$$
c_{\mathrm{st}} \approx\left(D^{2}-D\right)^{-1} \sqrt{D / 2}
$$

Thus the recommended value for the stirring coupling coefficient is 0.5 and $\sqrt{6} / 12 \approx 0.2$ for $D=2$ and $D=3$, respectively. Other criteria for the choice of the coupling coefficient are discussed later.

For the Nosé-Hoover and the fp-thermostats the change of the angular momentum reads

$$
\begin{aligned}
\dot{\boldsymbol{L}}= & -\left(v_{\mathrm{NH}} \alpha_{\mathrm{NH}}+\left(c_{\mathrm{fp}} / T\right) \boldsymbol{F} \cdot \boldsymbol{p} \alpha_{\mathrm{fp}}\right) \boldsymbol{L} \\
& +\left(c_{\mathrm{st}} / T\right) \boldsymbol{F} \cdot \boldsymbol{p} \boldsymbol{r} \times(\boldsymbol{W} \times \boldsymbol{p}) .
\end{aligned}
$$

Assuming, as above, $\dot{\boldsymbol{L}} \approx 0$, squaring and averaging the terms on the r.h.s. of the angular momentum balance leads to

$$
\begin{aligned}
& \left\langle\left(v_{\mathrm{NH}} \alpha_{\mathrm{NH}}+\left(c_{\mathrm{fp}} / T\right) \boldsymbol{F} \cdot \boldsymbol{p} \alpha_{\mathrm{fp}}\right)^{2} L^{2}\right\rangle \\
& \quad \approx c_{\mathrm{st}}^{2}\left\langle r^{2} F^{2}\right\rangle(D+2)(D-1)^{2} / D .
\end{aligned}
$$

Isotropy is assumed, e.g. $(\boldsymbol{F} \cdot \boldsymbol{p})^{2}$ is replaced by $F^{2} p^{2} / D$. For the canonical velocity distribution considered now one has $\left\langle p^{2 \mathrm{n}}\right\rangle=D(D+2) \ldots(D+n-$ 1) $T^{\mathrm{n}}$. Due to $\left\langle\alpha_{\mathrm{NH}}^{2}\right\rangle=\left\langle\alpha_{\mathrm{fp}}^{2}\right\rangle=1$ and $\left\langle\alpha_{\mathrm{NH}} \alpha_{\mathrm{fp}}\right\rangle=0$, and assuming that the averages of the products $F^{2} L^{2}$ can be factorized, one obtains

$$
\begin{aligned}
& v_{\mathrm{NH}}^{2}+c_{\mathrm{fp}}^{2}\left\langle F^{2}\right\rangle / T \approx c_{\mathrm{st}}^{2}\left(\left\langle r^{2} F^{2}\right\rangle /\left\langle r^{2}\right\rangle\right) \\
& \cdot(D+2)(D-1)^{2} /(2 T) .
\end{aligned}
$$

In equilibrium, $\left\langle F^{2}\right\rangle / T=\langle\Delta \Phi\rangle=D \omega_{\mathrm{E}}^{2}$ holds true where $\omega_{\mathrm{E}}$ is the Einstein frequency.

The recommended value for $c_{\text {st }}$ depends on the values chosen for $v_{\mathrm{NH}}$ and $c_{\mathrm{fp}}$. For the nonlinear elastic dumbbell considered here one has $\left\langle r^{2} F^{2}\right\rangle \approx\left\langle r^{2}\right\rangle\left\langle F^{2}\right\rangle=\left\langle r^{2}\right\rangle\langle\Delta \Phi\rangle T$ and $\langle\Delta \Phi\rangle \approx 6$. Then, for $D=2$, the relation given above implies $c_{\mathrm{st}} \approx\left(v_{\mathrm{NH}}^{2} / 12+c_{\mathrm{fp}}^{2} / 2\right)^{1 / 2}$. In particular, for $v_{\mathrm{NH}}=1$, $\sqrt{3}, 3,10$ on has $c_{\mathrm{st}}=0.289,0.5,0.866,2.887$ and $c_{\mathrm{st}}=0.764,0.886,1.118,2.972$ when $c_{\mathrm{fp}}$ is put equal to 0 and 1 , respectively. These and other values of the coupling coefficients have been tested, some results are presented next.

\section{Equilibrium Test Calculations}

\subsection{Model Potentials}

Test calculations were performed for the harmonic oscillator with the potential function $\Phi=r^{2} / 2$. The main attention is focussed on a nonlinear dumbbell model used previously. It intendes to mimic the dynamics and the shape of a polymer molecule by that of a particle at position $\boldsymbol{r}$ where $\boldsymbol{r}=0$ corresponds to the center of mass of the polymer molecule. The force $\boldsymbol{F}$ acting on this particle can be chosen such that the time average of $r^{2}$ coincides with the mean square radius of gyration of the polymer coil in equilibrium. In particular, the force is chosen as

$$
\boldsymbol{F}=-\boldsymbol{r} r^{-1} \boldsymbol{\Phi}^{\prime},-\boldsymbol{\Phi}^{\prime}=r^{-3}-r^{3}
$$

where $\Phi^{\prime}$ is the derivative of the potential function $\Phi(r)=(1 / 2) r^{-2}+(1 / 4) r^{4}-3 / 4$ with respect to $r$. The potential is of nonlinear elastic type with a minimum at $r=1$, in reduced units. The equilibrium radius of gyration $r_{0}$ is somewhat larger, depending on the temperature $T$. In particular one has $r_{0} \approx 1.02$ in two dimensions and for $T=0.1$, the temperature used in the previous studies. Here results will be presented for temperatures ranging from 0.05 to 2.0. The quantity $\langle\Delta \Phi\rangle$ which sets the scale for a frequency squared approaches, at low temperatures, the values 2 and 6 for the harmonic oscillator, in two dimensions, and for the dumbbell.

\subsection{Twirler and Gaussian Thermostat}

Some selected results from many test runs, performed with the Runge-Kutta version of NDSolve of Mathematica, are displayed in the following figures. In particular, Fig. 1 shows the orbit and the angular momentum versus time of the harmonic oscillator subjected to the Gaussian thermostat, with the temperature $T=1.0$, in reduced units. The upper and lower graph are for the stirring coupling coefficients $c_{\mathrm{st}}=0.0$ and 0.1 , repectively. The orbits are shown over 500 time units, the angular momentum over the last 180 time units only. Notice that the angular momentum does not 

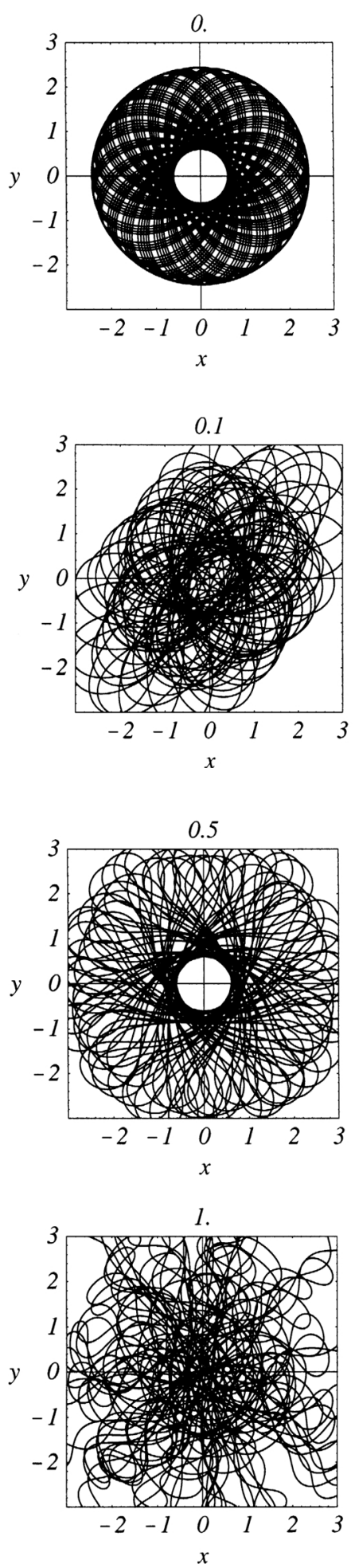
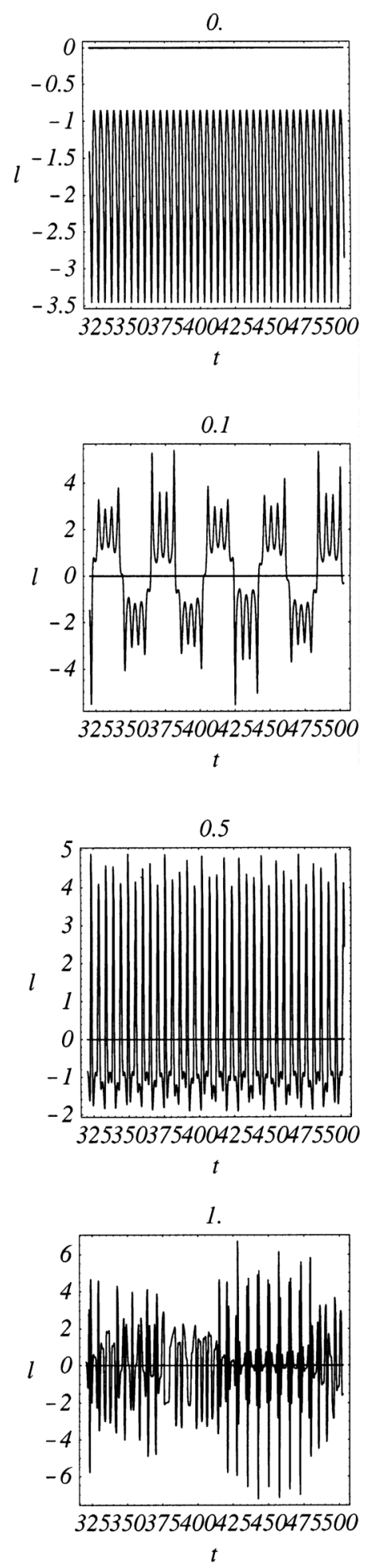

Fig. 1. The orbit and the angular momentum versus time of the harmonic oscillator subjected to the Gaussian thermostat. The temperature is $T=1.0 \mathrm{in}$ reduced units. The upper and lower graph are for the stirring coupling coefficients $c_{\mathrm{st}}=0.0$ and 0.1 , repectively. The orbits are shown over 500 time units, the angular momentum over the last 180 time units only.

Fig. 2. Same as the previous figure, but for the stirring coupling coefficients $c_{\mathrm{st}}=0.5$ and 1.0. 

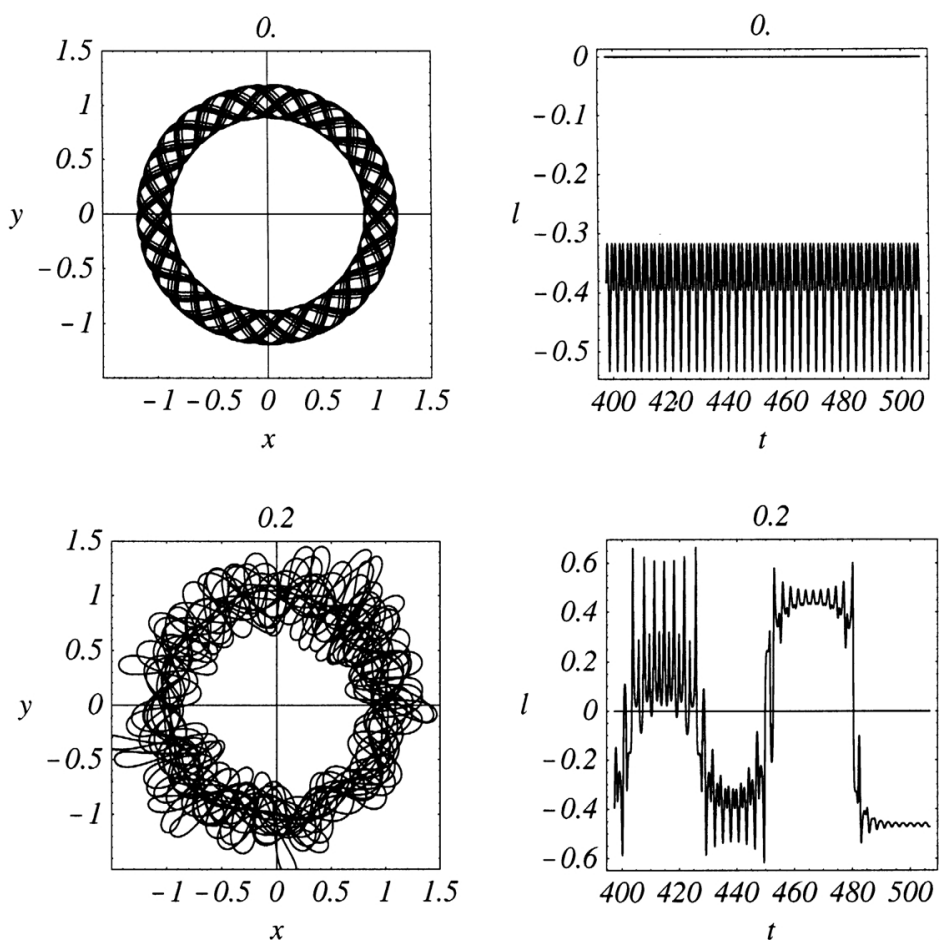

Fig. 3. The orbit and the angular momentum versus time of the nonlinear elastic dumbbell subjected to the Gaussian thermostat. The temperature is $T=0.1$ in reduced units. The upper and lower graph are for the stirring coupling coefficients $c_{\mathrm{st}}=0.0$ and 0.2 , repectively. The orbits are shown over about 500 time units, the angular momentum over the last 100 time units only.
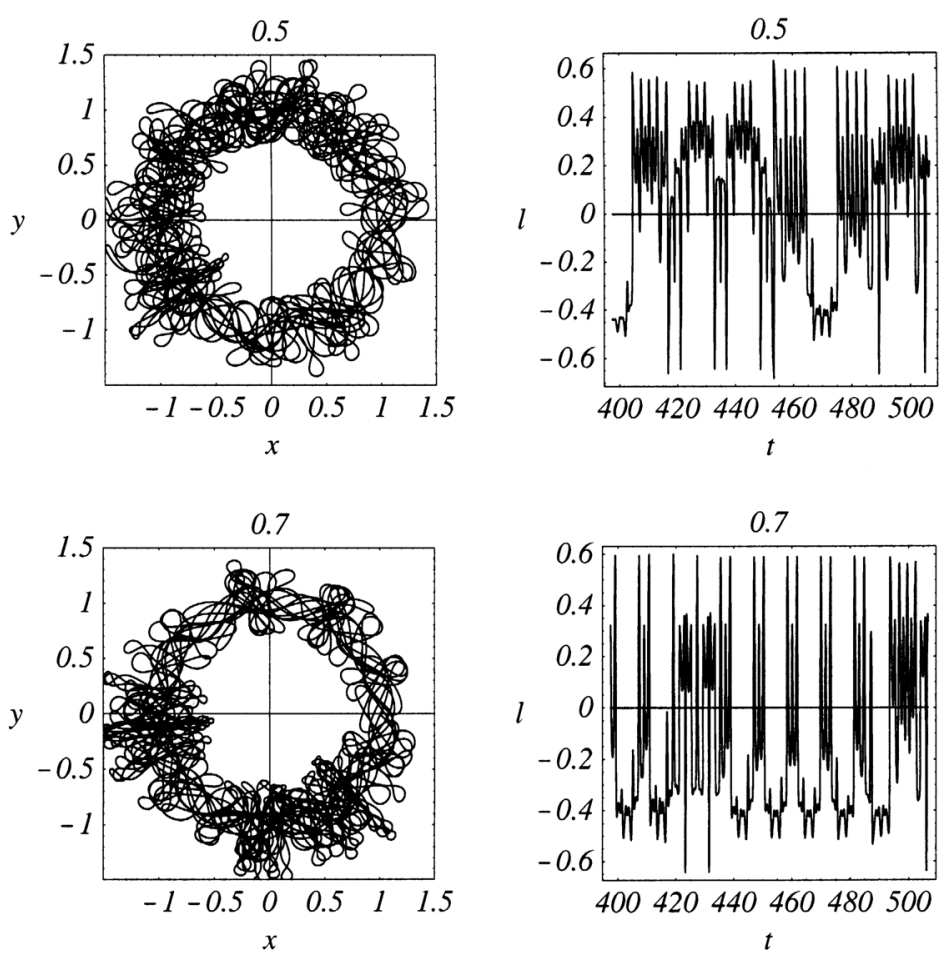

Fig. 4. Same as the previous figure, but for the stirring coupling coefficients $c_{\mathrm{st}}=0.5$ and 0.7 . 

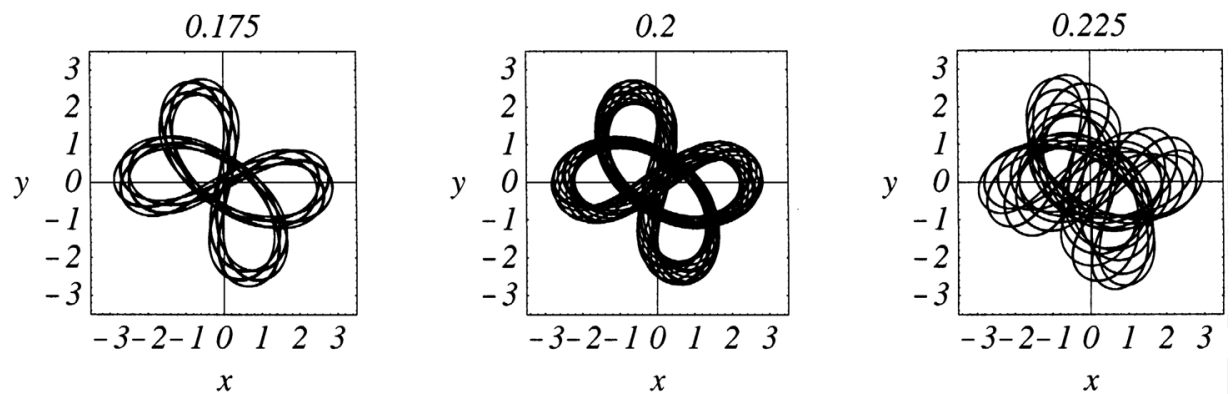

Fig. 5. The orbits of the harmonic oscillator subjected to the Gaussian thermostat and a twirler. The temperature is $T=1.0$ in reduced units. The coupling stirring coefficients are $c_{\mathrm{st}}=0.175,0.2$, and 0.225 , from left to right. The orbits are shown over about 300 time units.
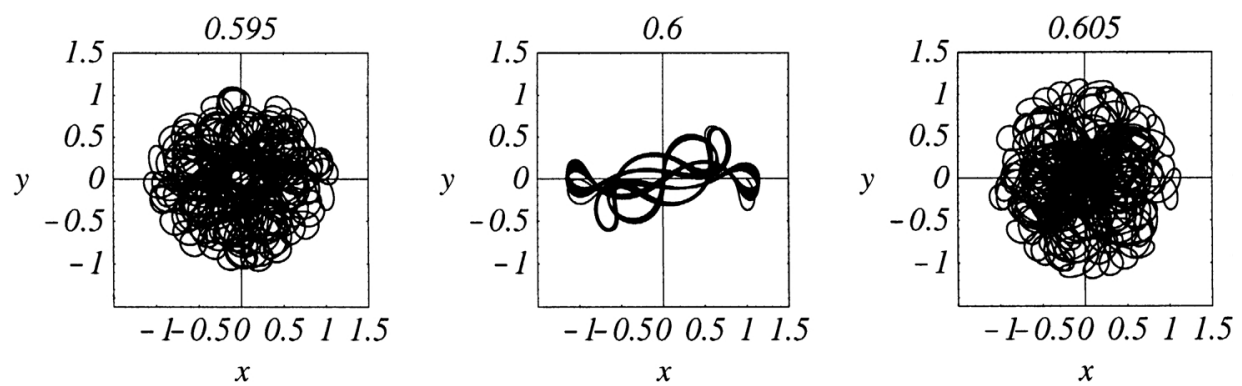

Fig. 6. Same as the previous figure, but now for the temperature $T=0.1$ and for the stirring coupling coefficients $C_{\mathrm{st}}=0.595$, 0.6 and 0.605 . The orbits are shown over about 600 time units.

change sign for the pure Gaussian thermostat corresponding to $c_{\mathrm{st}}=0.0$. It does change sign when the twirler is turned on, $i$. $e$. for $c_{\text {st }} \neq 0.0$. Analogous results for the stirring coupling coefficients $c_{\mathrm{st}}=0.5$ and 1.0 are depicted in Figure 2. Corresponding graphs for the orbit and the angular momentum versus time of the nonlinear elastic dumbbell subjected to the Gaussian thermostat, with the temperature is $T=0.1$, in reduced units, are shown in Figure 3. The upper and lower graph are for the stirring coupling coefficients $c_{\text {st }}=0.0$ and 0.2 , repectively. The orbits are shown over about 500 time units, the angular momentum over the last 100 time units only. Similar results for $c_{\text {st }}=0.5$ and 0.7 are presented in Figure 4. The initial conditions chosen were $x=1.0, y=0.0, v_{x}=\sqrt{T}$, and $v_{y}=-\sqrt{T}$, both for the harmonic oscillator and for the dumbbell.

In general, the twirler induces rather irregular motions and the angular momentum alternates around zero for nonzero stirring coupling coefficient, tested in the range 0.01 to 2.0. For certain values of the coupling coefficient $c_{\mathrm{st}}$, however, periodic orbits are observed, which look rather intriguing. Examples are shown for the harmonic oscillator in Fig. 5 and Fig. 6 where the temperature is 1.0 and 0.1 , respectively. In the first case, there occurs a double-brezel like structure in a relatively wide interval of coupling coefficients around 0.2 . In the second case, one observes a more complex orbit in very narrow range of values of $c_{\text {st }}$ around 0.6. Plots of the angular momentum do not easily reveal characteristic differences between the irregular and the periodic orbits. Of course, quantities which are sensitive to the "shape" of the orbit like the components $2 x y$ or $x^{2}-y^{2}$ of the gyration tensor are noticeably different for periodic and for irregular orbits.

To test the quality of the twirler, the average of the angular momentum $l_{z}$ and of its square, more specifically, $\left\langle l_{z}\right\rangle$ in units of $\sqrt{T G_{r}}$, and $\left\langle l_{z}^{2}\right\rangle$ divided by its equilibrium value $T G_{r}$ minus 1 , where $G_{r}=\left\langle r^{2}\right\rangle=$ $\left\langle x^{2}+y^{2}\right\rangle$ is the mean square radius, are plotted in the upper graph of Fig. 7 as functions of the stirring coupling coefficient $c_{\text {st }}$. The data are extracted for the last 300 time units of a total of about 350 . The lower graph shows $\left\langle x^{2}-y^{2}\right\rangle$ and $\langle 2 x y\rangle$, divided by $G_{r}$. For a good twirler, all quantities displayed in Fig. 7 should be close to zero. A good choice for the coupling coefficient is $c_{\mathrm{st}}=0.525$ where one has $\left\langle l_{z}\right\rangle / \sqrt{T G_{r}}=-0.0023,\left\langle l_{z}^{2}\right\rangle /\left(T G_{r}\right)-1=-0.0056$, $\langle 2 x y\rangle / G_{r}=0.00025,\left\langle x^{2}-y^{2}\right\rangle / G_{r}=-0.0041$. Aver- 

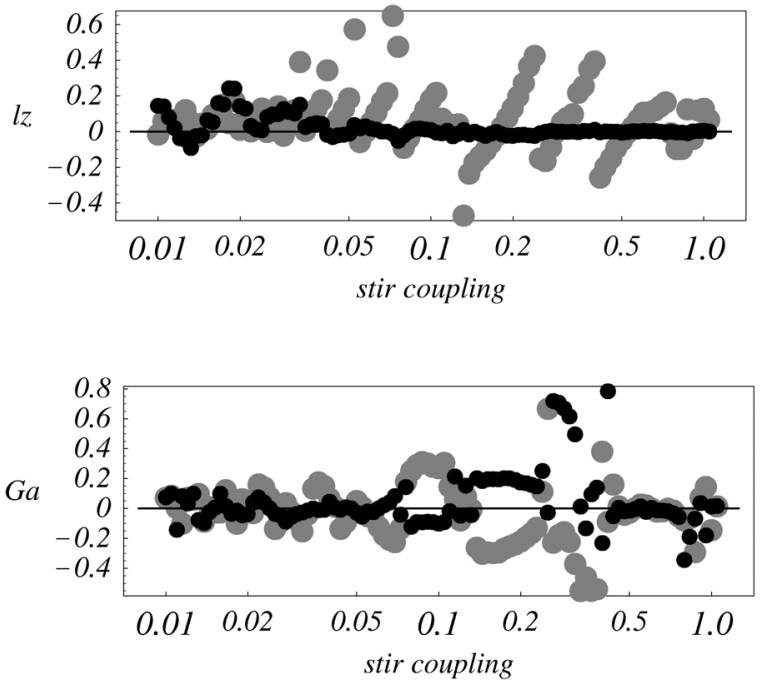

Fig. 7. The upper graph shows the angular momentum (black dots) and the square of the angular momentum (gray circles), the lower one the components of the gyration tensor characterizing its anisotropy, viz. $x^{2}-y^{2}$ (black dots) and $2 x y$ (gray circles) versus the stirring coupling coefficient, for the harmonic oscillator subjected to the Gaussian thermostat $(T=1)$ and the twirler. For the scaling of these quantities see the text.

aged over the last 3000 time units of a total of about 3500, one finds $\left\langle l_{z}\right\rangle / \sqrt{T G_{r}}=-0.00004,\left\langle l_{z}^{2}\right\rangle /\left(T G_{r}\right)-$ $1=0.0079,\langle 2 x y\rangle / G_{r}=-0.00046,\left\langle x^{2}-y^{2}\right\rangle / G_{r}=$ -0.00006 . Thus these quantities are practically zero, within computational accuracy, as expected for a harmonic oscillator in thermal equilibrium. In both cases the initial conditions were $x=\sqrt{2 T}, y=0.0, v_{x}=$ $\sqrt{T}$, and $v_{y}=-\sqrt{T}$. The data were extracted at 1000 or 10000 times seperated by the time interval $\delta t=$ $0.1 \pi / \sqrt{T}$, corresponding to $\delta t=0.314159$ for $T=1$.

The square root of the mean square radius, however, is larger than its equilibrium value by a factor of about 1.4. For the pure Gaussian thermostat $\left(c_{\mathrm{st}}=0\right)$, this factor is 1.15 . With the help of an additional configurational thermostat the mean square radius can be made to approach its equilibrium value. Then, however, $\left\langle l_{z}^{2}\right\rangle$ deviates more from its equilibrium value. No systematic search for the optimum values of $c_{\mathrm{st}}$ and of the effective relaxation time $\tau_{\mathrm{cf}}$, cf. (9), was conducted for the twirler combined with the Gaussian and configurational thermostats.

The dependence of the effect of the twirler on the corresponding averages for the nonlinear elastic dumbbell was analyzed in a similar fashion. The initial conditions were $x=1.0, y=0.0, v_{x}=\sqrt{T}$, and $v_{y}=-\sqrt{T}$.
Table 1. Averages of characteristic quantities, evaluated for the nonlinear elastic dumbbell, presented for selected values of the coupling coefficient $c_{\mathrm{st}}$.

\begin{tabular}{lccrc}
\hline$c_{\mathrm{st}}$ & $\left\langle l_{z}\right\rangle / \sqrt{T G_{r}}$ & $\left\langle l_{z}^{2}\right\rangle /\left(T G_{r}\right)-1$ & $\langle 2 x y\rangle / G_{r}$ & $\left\langle x^{2}-y^{2}\right\rangle / G_{r}$ \\
\hline 0.15 & 0.0096 & -0.023 & 0.022 & 0.019 \\
0.17 & -0.0060 & -0.002 & -0.005 & 0.002 \\
0.25 & 0.0163 & 0.074 & -0.009 & -0.021 \\
0.35 & 0.0602 & -0.004 & 0.038 & 0.030 \\
0.48 & -0.0201 & 0.002 & 0.057 & 0.003 \\
0.50 & -0.0698 & -0.047 & -0.031 & -0.058 \\
0.56 & -0.0566 & -0.011 & -0.037 & 0.067 \\
\hline
\end{tabular}

Averages were evaluted over the last 1000 time units of a total of about 1100 . More precisely, the data were extracted at 1000 times separated by the time interval $\delta t=0.1 \pi / \sqrt{T}$, corresponding to $\delta t=0.993459$ for $T=0.1$. Graphs corresponding to those shown in Fig. 7 are not displayed for sake of brevity. Good results are obtained for specific values of the coupling coefficient $c_{\mathrm{st}}$ only, some selected data are listed in Table 1. Here, a good recommendation for the choice of the coupling coefficient is $c_{\text {st }}=0.17$. The tempting value $c_{\mathrm{st}}=1.0$ should not be chosen since there occur peculiar periodic orbits for coupling coefficients in the vicinity of 1.0 leading to nonzero averages for the angular momentum.

The problem with an increased value of $G_{r}=\left\langle r^{2}\right\rangle$ is not so severe for the nonlinear elastic dumbbell due to the existence of a minimum of the potential at a finite distance. To be more specific, the square root of the mean square radius is equal to 1.04 and 1.06 for the pure Gaussian thermostat with $c_{\mathrm{st}}=0$ and the additional twirler with $c_{\mathrm{st}}=0.17$. The equilibrium value is 1.01 .

\subsection{Twirler, Nosé-Hoover and Force-Momentum Thermostats}

Numerous calculations which test the effectiveness of the twirler have been performed for the nonlinear elastic dumbbell subjected to the Nosé-Hoover and force-momentum thermostats. The temperature prescribed was $T=0.1$. For the Nosé-Hoover relaxation frequency $v_{\mathrm{NH}}$ and the force-momentum coupling coefficient $c_{\mathrm{fp}}$, several values were chosen, and the twirler coupling coefficient $c_{\mathrm{st}}$ was varied systematically between 0.01 and 1.0, in some cases up to 3.0, in steps of 0.01 . In some selected intervals, steps of 0.001 were taken. Averages of the characteristic quantities

$$
\begin{aligned}
& \left\langle l_{z}\right\rangle^{*}=\left\langle l_{z}\right\rangle / \sqrt{T G_{r}},\left\langle l_{z}^{2}\right\rangle^{*}=\left\langle l_{z}^{2}\right\rangle /\left(T G_{r}\right)-1, \\
& 2 G_{+}^{*}=\langle 2 x y\rangle / G_{r}, G_{-}^{*}=\left\langle x^{2}-y^{2}\right\rangle / G_{r},
\end{aligned}
$$




\begin{tabular}{lllrrrrrrr}
\hline$v_{\mathrm{NH}}$ & $c_{\mathrm{fp}}$ & \multicolumn{1}{c}{$c_{\mathrm{st}}$} & \multicolumn{1}{c}{$\left\langle l_{z}\right\rangle^{*}$} & \multicolumn{1}{c}{$\left\langle l_{z}^{2}\right\rangle^{*}$} & \multicolumn{1}{c}{$2 G_{+}^{*}$} & \multicolumn{1}{c}{$G_{-}^{*}$} & \multicolumn{1}{c}{$G_{r}$} & \multicolumn{1}{r}{$\left\langle p^{2}\right\rangle^{*}$} & \multicolumn{1}{r}{$\left\langle p^{4}\right\rangle^{*}$} \\
\hline 10.0 & 0.0 & 0.12 & 0.0079 & -0.218 & 0.017 & 0.030 & 1.066 & 0.039 & 0.005 \\
10.0 & 0.0 & 0.71 & 0.0003 & 0.103 & -0.008 & 0.029 & 1.059 & -0.038 & 0.015 \\
10.0 & 0.0 & $2.884^{*}$ & 0.0061 & -0.012 & -0.093 & 0.031 & 1.070 & 0.012 & -0.077 \\
10.0 & 1.0 & 0.06 & -0.0061 & -0.100 & 0.004 & 0.006 & 1.098 & 0.026 & 0.232 \\
10.0 & 1.0 & 0.96 & 0.0015 & -0.100 & -0.015 & -0.067 & 1.055 & -0.043 & 0.014 \\
10.0 & 1.0 & $2.97^{*}$ & -0.0053 & 0.045 & -0.054 & 0.082 & 1.062 & -0.029 & -0.034 \\
3.0 & 0.0 & 0.09 & -0.0011 & 0.119 & -0.003 & 0.002 & 1.069 & -0.055 & -0.211 \\
3.0 & 0.0 & 0.56 & 0.0080 & 0.081 & -0.012 & 0.001 & 1.062 & -0.009 & -0.036 \\
3.0 & 0.0 & $0.86^{*}$ & -0.0033 & -0.038 & -0.062 & 0.119 & 1.064 & -0.083 & -0.081 \\
3.0 & 1.0 & 0.35 & 0.0004 & 0.006 & -0.076 & -0.043 & 1.061 & 0.005 & -0.046 \\
3.0 & 1.0 & 0.46 & -0.0023 & -0.001 & -0.032 & 0.040 & 1.062 & 0.009 & -0.033 \\
3.0 & 1.0 & $1.11^{*}$ & -0.0043 & -0.006 & 0.068 & 0.106 & 1.054 & 0.013 & 0.097 \\
$\sqrt{3}$ & 0.0 & 0.13 & -0.0072 & -0.053 & -0.050 & 0.007 & 1.080 & -0.001 & -0.019 \\
$\sqrt{3}$ & 0.0 & 0.24 & -0.0051 & -0.111 & 0.054 & 0.011 & 1.065 & 0.001 & -0.042 \\
$\sqrt{3}$ & 0.0 & $0.50^{*}$ & 0.0069 & 0.120 & -0.032 & -0.078 & 1.052 & 0.015 & 0.136 \\
$\sqrt{3}$ & 0.0 & 0.78 & -0.0003 & 0.021 & -0.062 & -0.016 & 1.083 & -0.003 & -0.018 \\
$\sqrt{3}$ & $\sqrt{2} / 4$ & $0.54^{*}$ & -0.0024 & 0.159 & -0.081 & -0.033 & 1.042 & -0.009 & -0.064 \\
$\sqrt{3}$ & 1.0 & 0.19 & 0.0087 & 0.075 & 0.042 & -0.014 & 1.063 & 0.015 & 0.025 \\
$\sqrt{3}$ & 1.0 & $0.88^{*}$ & 0.0098 & 0.013 & 0.038 & 0.017 & 1.058 & -0.013 & -0.069 \\
1.0 & 0.0 & 0.86 & 0.0051 & 0.024 & 0.002 & -0.031 & 1.070 & 0.003 & -0.021 \\
1.0 & 1.0 & 0.26 & -0.0001 & -0.134 & -0.004 & 0.014 & 1.072 & 0.025 & -0.051 \\
1.0 & 1.0 & 0.40 & 0.0089 & 0.062 & 0.007 & -0.006 & 1.073 & 0.026 & -0.065 \\
1.0 & 1.0 & 0.51 & 0.0004 & -0.004 & -0.010 & -0.053 & 1.059 & -0.033 & 0.047 \\
1.0 & 1.0 & $0.76^{*}$ & -0.0070 & -0.155 & -0.082 & 0.061 & 1.066 & -0.026 & -0.165 \\
0.0 & 1.0 & 0.30 & 0.0053 & -0.035 & -0.065 & -0.026 & 1.092 & 0.03 & -0.293 \\
\hline & & & & & & & & &
\end{tabular}

Table 2. Averages of characteristic quantities, evaluated for the nonlinear elastic dumbbell for $T=0.1$, presented for selected values of the relaxation frequency and coupling coefficients $v_{\mathrm{NH}}, c_{\mathrm{fp}}$, and $c_{\mathrm{st}}$.
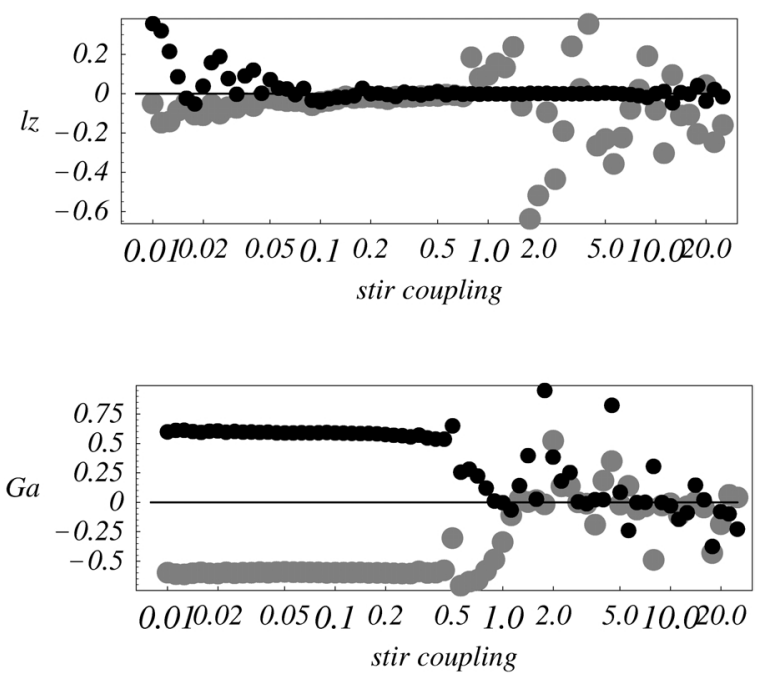

Fig. 8. The upper graph shows the angular momentum (black dots) and the square of the angular momentum (gray circles), the lower one the components of the gyration tensor characterizing its anisotropy, viz. $x^{2}-y^{2}$ (black dots) and $2 x y$ (gray circles) versus the stirring coupling coefficient, for the adiabatic harmonic oscillator with the energy $E=2.0$, subjected to the twirler. For the scaling of these quantities see the text.

and

$\left\langle p^{2}\right\rangle^{*}=\left\langle p^{2}\right\rangle /(2 T)-1,\left\langle p^{4}\right\rangle^{*}=\left\langle p^{4}\right\rangle /\left(8 T^{2}\right)-1$, were computed over the last 1000 time units of a total of about 1100. The initial conditions were chosen and the extraction of the data was done just as in the case of the Gaussian thermostat discussed above. Some results for these averages which should be close to zero in thermal equilibrium are presented in Table 2. Also listed are values for $G_{r}=\left\langle x^{2}+y^{2}\right\rangle$ which should be close to 1.02. Data are shown for some of those coupling coefficients $c_{\text {st }}$ where the magnitude of the average scaled angular momentum $\left\langle l_{z}\right\rangle^{*}$ is less than $1 / 100$. The values of $c_{\mathrm{st}}$ marked by a star ${ }^{*}$ are close to those values recommended by the considerations given above. These recommended values give satisfactory results, however, there are combinations of relaxation frequency and coupling coefficients which seem to work better.

Notice that the last row of Table 2 shows data for the twirler combined with the force-momentum thermostat but with the Nosé-Hoover thermostat turned off. Remarkably enough, the average of $p^{2}$ is close to its equilibrium value for all $c_{\text {fp }}$ larger than 0.1 , whereas the average of $p^{4}$ is systematically too small.

A word of caution is in order. The length of Table 2 seems to imply that practically any value of $c_{\text {st }}$ can be used. However, for many $c_{\text {st }}$, one or more of the magnitude of averages of quantities which should be close to zero are larger than $1 / 10$. In some cases periodic 

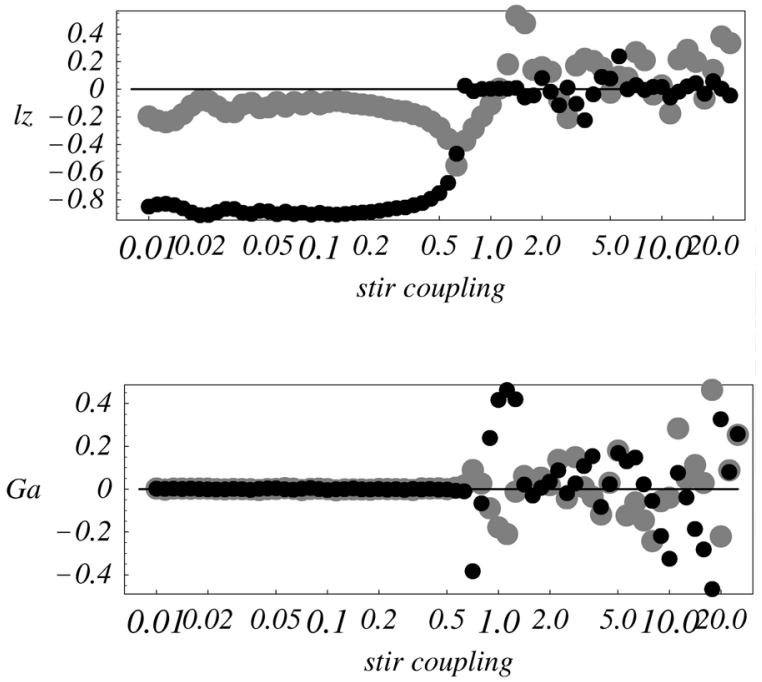

Fig. 9. The upper graph shows the angular momentum (black dots) and the square of the angular momentum (gray circles), the lower one the components of the gyration tensor characterizing its anisotropy, viz. $x^{2}-y^{2}$ (black dots) and $2 x y$ (gray circles) versus the stirring coupling coefficient, for the nonlinear elastic dumbbell with the energy $E=0.15$, subjected to the twirler. For the scaling of these quantities see the text.

orbits occur. For $v_{\mathrm{NH}}=1.0$ and $c_{\mathrm{fp}}=0$, the angular momentum does not average to zero at all values of $c_{\mathrm{st}}$ less than 0.8 . For all other combinations of $v_{\mathrm{NH}}$ and $c_{\mathrm{fp}}$ studied relatively large values of the average angular momentum occur for certain values of $c_{\mathrm{st}}$ in a rather irregular fashion. So before the twirler and the thermostats are applied to the study of specific problems like the influence of additional forces or the effects of a flow field, the equilibrium behavior has to be tested.

It is also of interest to study the effect of the twirler for adiabatic systems where no thermostat is applied. Some results are presented in the appendix and in Figs. 8 and 9.

\section{Rotational Diffusion}

The rotational diffusion coefficient can be inferred from the mean square angular displacement via an Einstein relation. The angular displacement $\Delta \varphi$ is the time integral of the angular velocity $\omega(t)=l_{z}(t) / G_{r}(t)$ where $G_{r}$ is the relevant moment of inertia. The time integration is approximated by evaluating the angular velocity in time steps seperated by $\delta t$, by performing the appropriate summation and multiplication by $\delta t$. The value $\delta t=1.0$ was chosen in the calculations pre-

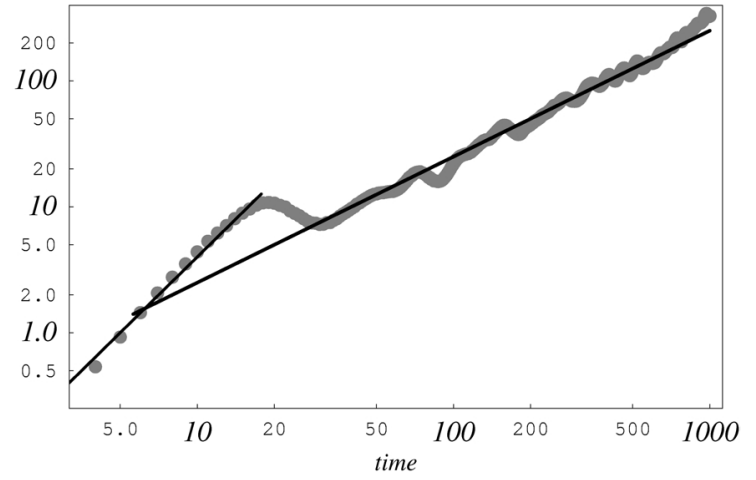

Fig. 10. The mean square angular displacement, divided by 2 , as function of the time for a dumbbell subjected to the Gaussian thermostat with $T=0.1$ and a twirler with the coupling coefficient $c_{\mathrm{st}}=0.17$. The thin and thick lines have slopes 2 and 1 , respectively, the rotational diffusion coefficient is $\bar{D}_{r}=0.25$.

sented next. The square of the angular displacenent is averaged over different runs with randomly chosen initial conditions. A time dependent rotational diffusion coefficent $D_{r}(t)$ is defined via the relation

$$
\left\langle(\Delta \varphi)^{2}\right\rangle=2 D_{r}(t) t
$$

The "true" rotational coefficient $\bar{D}_{r}$ is obtained when $D_{r}(t)$ reaches a plateau value at large times. For the time reversal chaotic dynamics analysed here, one has to test whether such a diffusive behavior exists at all. It indeed seems to be the case for the examples studied here. In Fig. 10 the mean square angular displacement, divided by 2 , is displayed as function of the time for the nonlinear elastic dumbbell subjected to the Gaussian thermostat with $T=0.1$ and a twirler where the coupling coefficient is $c_{\mathrm{st}}=0.17$. The thin and thick lines have slopes 2 and 1 , respectively, the rotational diffusion coefficient, inferred from the average between $t=500$ and $t=1000$, is $\overline{D_{r}}=0.25$. The average $\langle\ldots\rangle$ is based on 100 different initial conditions with the initial positions and velocities $x=\cos \left(2 \pi R_{\text {pos }}\right)$, $y=\sin \left(2 \pi R_{\mathrm{pos}}\right), v_{x}=\sqrt{2 T} \cos \left(2 \pi R_{\mathrm{vel}}\right), v_{y}=\sqrt{2 T}=$ $\sin \left(2 \pi R_{\mathrm{vel}}\right)$, where $R_{\mathrm{pos}}$ and $R_{\mathrm{vel}}$ are random numbers between 0 and 1 generated, within the Mathematica program, by Random[] with SeedRandom [143]. In Figs. 11 and 12 the rotational diffusion coefficient $D_{r}(t)$ is shown as function of the time $t$ for the nonlinear elastic dumbbell subjected to the Gaussian thermostat, now for $T=0.05$, and a twirler with the coupling coefficients $c_{\mathrm{st}}=0.17$ and $c_{\mathrm{st}}=0.5$, repectively. The horizontal lines correspond to the averages evalu- 


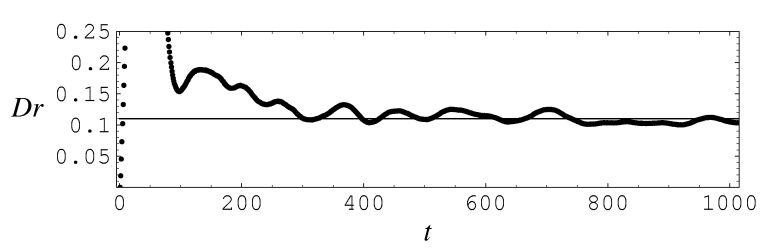

Fig. 11. The rotational diffusion coefficient $D_{r}(t)$ as function of the time $t$ for a dumbbell subjected to the Gaussian thermostat with $T=0.05$ and a twirler with the coupling coefficient $c_{\mathrm{st}}=0.17$. The horizontal line corresponds to the average over the last 500 time units, viz.: $\bar{D}_{r}=0.11$.

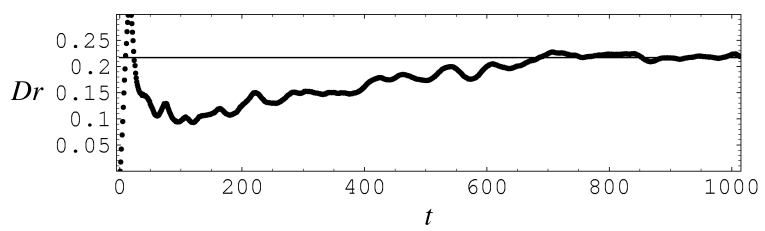

Fig. 12. The rotational diffusion coefficient $D_{r}(t)$ as function of the time $t$ for a dumbbell subjected to the Gaussian thermostat with $T=0.05$ and a twirler with the coupling coefficient $c_{\mathrm{st}}=0.5$. The horizontal line corresponds to the average over the last 500 time units, viz.: $\bar{D}_{r}=0.21$.

ated over the last 500 time units, viz.: $\bar{D}_{r}=0.11$ and $\bar{D}_{r}=0.21$. The plateau value can be approached from above, as in Fig. 11 or from below, cf. Figure 12.

As a side remark which is of interest for further applications, it is mentioned that, for $T=0.1$, the average based on just 10 runs with the initial positions and velocities $x=1.0, y=0.0$, and $v_{x}=\sqrt{2 T} \cos \left(2 \pi R_{\mathrm{vel}}\right)$, $v_{y}=\sqrt{2 T} \sin \left(2 \pi R_{\mathrm{vel}}\right)$, where the $R_{\mathrm{vel}}$ are random numbers generated with the seed mentioned above, yields practically the same rotational diffusion coefficients $\bar{D}_{r}$.

\section{Shear-Induced Angular Velocity}

The influence of a shear flow on the angular velocity of a polymer chain molecule has recently been analysed in non-equilibrium molecular dynamics (NEMD) computer simulations of polymer solutions [11]. The basic features are already seen in simple model calculations for thermostated nonlinear elastic dumbbells. In [10], Gaussian, Nosé-Hoover and configurational thermostats were used. These thermostats alone do not randomize the direction of the rotational velocity. Thus in order to detect the influence of the shear on the angular velocity for shear rates that are small compared with the thermal angular velocity, the difference in the behavior of two dumbbells was analysed, which started from equal initial positions but with opposite direc- tions of their angular velocities. Here test results are presented for a single dumbbell subjected to a shear flow; the Gaussian isokinetic thermostat and a twirler with $c_{\mathrm{st}}=0.5$ are applied.

To be more specific, it is assumed that the dumbbell feels a pseudo-friction force $-\zeta(\dot{r}-\boldsymbol{v}(\boldsymbol{r}))$ where $\boldsymbol{v}(\boldsymbol{r}))$ is the flow velocity of the fluid which, for simplicity, is assumed to be not affected by the presence of the dumbbell, i.e. the flow acts like an "external" field. The quantity $\zeta$ is the pseudo-friction coefficient of the Gaussian thermostat. In the following it is convenient to introduce the "momentum" variable

$$
\boldsymbol{p}=\frac{\mathrm{d} \boldsymbol{r}}{\mathrm{d} t}-\boldsymbol{v}(\boldsymbol{r})
$$

which is the peculiar velocity.

Next, the special case of a plane Couette flow is considered with the velocity given by $\boldsymbol{v}(\boldsymbol{r})=\gamma y \boldsymbol{e}^{x}$, where $\boldsymbol{e}^{x}$ is a unit vector in $x$-direction and $\gamma=\partial v_{x} / \partial y=$ const is the shear rate. Furthermore, as before, the motion of the particle is restricted to the $x y$-plane. Then, appart from the new terms involving $W=W_{z}$, the equations of motion correspond to the (two-dimensional version) of the "SLLOD" algorithm used in NEMD simulation studies of the viscous properties of fluids [3]:

$$
\begin{aligned}
& \frac{\mathrm{d} x}{\mathrm{~d} t}=p_{x}+\gamma y, \quad \frac{\mathrm{d} y}{\mathrm{~d} t}=p_{y} \\
& \frac{\mathrm{d} p_{x}}{\mathrm{~d} t}=F_{x}-\zeta p_{x}-\left(c_{\mathrm{st}} / T\right) \boldsymbol{F} \cdot \boldsymbol{p} W p_{y}-\gamma p_{y} \\
& \frac{\mathrm{d} p_{y}}{\mathrm{~d} t}=F_{y}-\zeta p_{y}+\left(c_{\mathrm{st}} / T\right) \boldsymbol{F} \cdot \boldsymbol{p} W p_{x}
\end{aligned}
$$

The expression for the coefficient $\zeta$ now is

$$
\zeta=\left(\boldsymbol{p} \cdot \boldsymbol{F}-\gamma p_{x} p_{y}\right) / p^{2}
$$

which guarantees that $p^{2}=2 T=$ const. The value of the temperature $T$ is fixed by the initial condition. The time average of $\zeta$ vanishes for $\gamma=0$. The same applies to the variable $W$. Furthermore (6) reduces to

$$
\frac{\mathrm{d} W}{\mathrm{~d} t}=-\left(c_{\mathrm{st}} / T\right)\left(F_{x} p_{y}-F_{y} p_{x}\right)
$$

The relation between the shear-induced angular velocity and the influence of the shear flow on the shape of the orbit, as derived in $[10,11]$ is based on the angular momentum balance and the assumption that the 
time change of the angular approaches zero when averaged over long times. For the plane Couette geometry considered here, this leads to

$$
\left\langle L_{z}\right\rangle=-\gamma m\left\langle G_{y y}\right\rangle .
$$

The angular bracket indicates the time average. The angular velocity $\bar{\omega}$ is defined as the ratio of $\langle\boldsymbol{L}\rangle$ and of the relevant component of the (time averaged) moment of inertia tensor, viz. $\left(G_{x x}+G_{y y}\right)$. Thus the expression for the angular velocity, inferred from the average angular momentum is

$$
\bar{\omega}=\frac{\left\langle L_{z}\right\rangle}{\left(\left\langle G_{x x}\right\rangle+\left\langle G_{y y}\right\rangle\right)} .
$$

The relation $(25)$ becomes

$$
\bar{\omega}=\bar{\omega}_{G},
$$

where the expression $\bar{\omega}_{G}$ is based on the shape of the orbit, used to model the geometry of the polymer coil, which is given by

$\bar{\omega}_{G}=-\gamma \frac{\left\langle G_{y y}\right\rangle}{\left\langle G_{x x}\right\rangle+\left\langle G_{y y}\right\rangle}=-\frac{\gamma}{2}\left(1-\frac{\left\langle G_{x x}\right\rangle-\left\langle G_{y y}\right\rangle}{\left\langle G_{x x}\right\rangle+\left\langle G_{y y}\right\rangle}\right)$.

In an undeformed equilibrium state, the coil is spherical, on average, then one has $\bar{\omega}_{G}=-\gamma / 2$. The same applies for small shear rates. At intermediate and at high shear rates, the polymer molecule is substanially deformed such that $G_{x x}>G_{y y}$, on average. This implies that the ratio $\Omega_{G}=\bar{\omega}_{G} /(-\gamma)$ becomes significantly smaller than $1 / 2$ which is its small shear rate limiting value. A relation of the form (27) between the rotational angular velocity $\bar{\omega}$ and the quantity $\bar{\omega}_{G}$ associated with the deformation of the chain molecule was first proposed by Cerf [18]. Non-equilibrium molecular dynamics (NEMD) computer simulations can and have provided [11] a test of this relation. Here, as in [10], a test is conducted for a simple two-dimensional dumbbell model. In Fig. 13, the angular velocity, divided by the negative shear rate, viz. $\bar{\omega} /(-\gamma)$ (black dots) and its geometric counterpart $\bar{\omega}_{G} /(-\gamma)$ (larger gray dots) are shown as functions of the shear rate for a dumbbell subjected to the Gaussian thermostat and a twirler with the coupling coefficient $c_{\mathrm{st}}=0.5$. The data were collected over $40 / \gamma$ time units and averaged over 10 runs with random initial conditions chosen as indicated at the end of the preceding

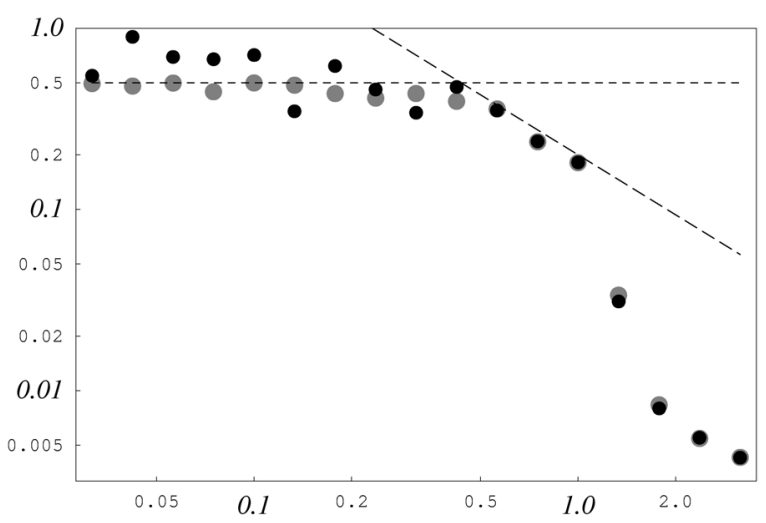

Fig. 13. The angular velocity, divided by the negative shear rate (black dots) and its geometric counterpart (larger gray dots) as functions of the shear rate for a dumbbell subjected to the Gaussian thermostat and a twirler with the coupling coefficient $c_{\mathrm{st}}=0.5$. The dashed horizontal line marks the value 0.5 expected for a solid-like rotation, the inclined dashed line corresponds to a power law with the exponent -1.1 .

section. The dashed horizontal line marks the value 0.5 expected for a solid-like rotation, the inclined dashed line corresponds to a power law with the exponent -1.1 . This value is close to that one found in NEMD simulations of polymer chains [11]. For $c_{\text {st }}=0.17 \mathrm{a}$ steeper decrease of the angular velocity, with the power law exponent -2.5 , is observed for high shear rates. The systematic study of the effects of the shear flow on the orbit and on long time averages for different coupling coefficients $c_{\mathrm{st}}$ is outside the scope of this article.

\section{Concluding Remarks}

In this article, it is demonstrated how the timereversible equations of motion can be coupled with an additional dynamical variable which obeys its own equation of change such that the angular momentum of a rotating particle is randomized in equilibrium. This twirler has been combined with the Gaussian, the Nosé-Hoover, the force-momentum and the configurational thermostats. Here, test calculations and applications to rotational diffusion and to the shear-flow induced modifications of the rotational motion and of the shape of the orbit were given for the two-dimensional isotropic harmonic oscillator and, in particular, for a nonlinear elastic dumbbell. Some results for the effect the adiabatic twirler are presented in the appendix.

Alternative time-reversible equations can be formulated when a method, introduced in [6] for the randomization of a unit vector ("spin"), is applied to the direc- 


\begin{tabular}{crcrccccc}
\hline$c_{\mathrm{st}}$ & \multicolumn{1}{c}{$\left\langle l_{z}\right\rangle^{*}$} & $\left\langle l_{z}^{2}\right\rangle^{*}$ & \multicolumn{1}{c}{$2 G_{+}^{*}$} & \multicolumn{1}{c}{$G_{-}^{*}$} & $G_{r} / 2$ & $r_{\mathrm{sh}}$ & $\left\langle E_{\mathrm{kin}}\right\rangle$ & $p_{4}$ \\
\hline 2.80 & 0.0004 & 0.743 & 0.001 & -0.005 & 1.000 & 0.007 & 1.003 & 0.702 \\
2.85 & -0.0002 & 0.889 & -0.003 & -0.006 & 1.002 & 0.012 & 1.002 & 0.681 \\
2.90 & -0.0001 & 0.977 & 0.003 & 0.003 & 1.002 & 0.021 & 1.003 & 0.665 \\
2.92 & 0.0004 & 1.006 & 0.004 & 0.002 & 1.000 & 0.009 & 1.005 & 0.659 \\
2.95 & 0.0001 & 1.045 & 0.046 & -0.056 & 1.003 & 0.009 & 1.003 & 0.653 \\
3.00 & 0.0002 & 1.098 & -0.009 & 0.001 & 1.003 & 0.025 & 1.004 & 0.643 \\
3.05 & -0.0006 & 1.135 & -0.006 & 0.005 & 1.005 & 0.023 & 1.003 & 0.635 \\
3.10 & 0.0007 & 1.188 & -0.009 & 0.008 & 1.004 & 0.025 & 1.004 & 0.628 \\
\hline & & & & & & & & \\
\hline$c_{\mathrm{st}}$ & \multicolumn{1}{c}{$\left\langle l_{z}\right\rangle^{*}$} & $\left\langle l_{z}^{2}\right\rangle^{*}$ & $2 G_{+}^{*}$ & $G_{-}^{*}$ & $G_{r} / 2$ & $r_{\mathrm{sh}}$ & $\left\langle E_{\mathrm{kin}}\right\rangle$ & $p_{4}$ \\
\hline 2.14 & 0.0038 & 1.104 & 0.017 & -0.052 & 1.071 & 0.065 & 0.106 & 0.581 \\
2.19 & -0.0093 & 1.171 & -0.047 & 0.023 & 1.078 & 0.094 & 0.110 & 0.559 \\
2.20 & 0.0010 & 1.073 & 0.046 & 0.026 & 1.067 & 0.185 & 0.105 & 0.583 \\
2.29 & -0.0071 & 1.035 & 0.078 & 0.087 & 1.060 & 0.142 & 0.099 & 0.608 \\
\hline
\end{tabular}

Table 3. Averages of characteristic quantities, evaluated for the adiabatic harmonic oscillator with $E=2.0$, presented for selected values of the coupling coefficient $c_{\mathrm{st}}$.

tion of the velocity. In that approach, which has been tested for a double dumbbell or trumbbell [18], one has to choose an anisotropic potential which imposes certain symmetry properties on the system which should be distinct from those inherent in the physical problem studied. The present method seems to be conceptionally simpler, nevertheless, a comparison between both methods should be performed.

The extension of test calculations and applications of the kind considered here to 3-dimensional rotations, as well as the study of trumbbells [19] and more complex molecular models are desireable. A further application is the analysis of the effect of confining walls on the rotation and, in particular, on the rotational diffusion, as observed in NMR-experiments [20].

\section{Acknowledgements}

This work has been conducted under the auspices of the Sonderforschungsbereich SFB 448 "Mesoskopisch strukturierte Verbundsysteme" of the Deutsche Forschungsgemeinschaft (DFG). Financial support is gratefully acknowledged. Furthermore, I thank Bill Hoover, Patrick Ilg, and Martin Kröger for helpful discussions.

\section{Appendix: Adiabatic Twirler}

It is also of interest to test the effect of the twirler for adiabatic systems where no thermostat is applied. Results for the harmonic oscillator with the initial conditions $x=\sqrt{2}, y=0.0, v_{x}=1$, and $v_{y}=-1$, which imply the inital kinetic energy $E_{\text {kin }}=1.0$ and the total energy $E=2.0$, are shown in Fig. 8 and in Table 3. Here the scaled variables

$$
\begin{aligned}
& \left\langle l_{z}\right\rangle^{*}=\left\langle l_{z}\right\rangle / \sqrt{\left\langle E_{\mathrm{kin}}\right\rangle G_{r} / 2}, \\
& \left\langle l_{z}^{2}\right\rangle^{*}=2\left\langle l_{z}^{2}\right\rangle /\left(\left\langle E_{\mathrm{kin}}\right\rangle G_{r}\right),
\end{aligned}
$$

and

$$
p_{4}=\left\langle p^{4}\right\rangle /\left(2\left\langle p^{2}\right\rangle^{2}\right)
$$

are used, for $G_{+}^{*}$ and $G_{-}^{*}$ see (16). Furthermore, $r_{\mathrm{sh}}=$ $\sqrt{\langle x\rangle^{2}+\langle y\rangle^{2}}$ is the shift of the "center of mass" of the orbit with respect to the origin. The angular momentum averages to zero for $c_{\text {st }}>0.1$, the quantities $G_{+}^{*}$ and $G_{-}^{*}$ characterizing the anisotropy of the orbit become close to zero for $c_{\mathrm{st}}>1.0$. A good performance of the twirler is found for $c_{\mathrm{st}} \approx 3$, cf. Table 3 . Here the average of the angular momentum and $G_{+}^{*}, G_{-}^{*}$ are rather small. The average kinetic energy and the mean square radius $\left\langle r^{2}\right\rangle=G_{r}$ are just slightly larger than their initial values. For a particle at distance $r$ from the center and with the tangential momentum $p_{\text {tan }}$, the instantaneous Lyapunov coefficients are $0, \pm i$, $(1 / 2)\left[\left(c_{\mathrm{st}} / T\right) w r p_{\tan } \pm \sqrt{\left(c_{\mathrm{st}} / T\right)^{2} w^{2} r^{2} p_{\mathrm{tan}}^{2}-4}\right]$. The Lyapunov exponents $\lambda$ are the averages of these quantities. The estimate where the average of the square root is replaced by the square root of averages, with $\langle w\rangle=0,\left\langle w^{2}\right\rangle=1,\left\langle p_{\tan }^{2}\right\rangle=T,\left\langle r^{2}\right\rangle=2 T$, leads to $\lambda=0, \pm i, \pm \sqrt{c_{\mathrm{st}}^{2} / 2-1}$. Thus one of the Lyapunov exponents has a real part larger than zero for $c_{\mathrm{st}}>\sqrt{2}$ and the system behaves in a chaotic manner. These considerations, however, provide just a crude estimate and do not replace the proper calculation of Lyapunov exponents which is outside the scope of the current investigation. It should be mentioned that some bewildering (periodic) orbits are observed for special values of the coupling coefficient $c_{\text {st }}$, cf. Figure 14. Notice that several of these orbits, at least over the 200 time units shown, have broken the rotational symmetry of the interaction potential.

Corresponding results for the nonlinear elastic dumbbell with the initial conditions $x=1.0, y=$ 

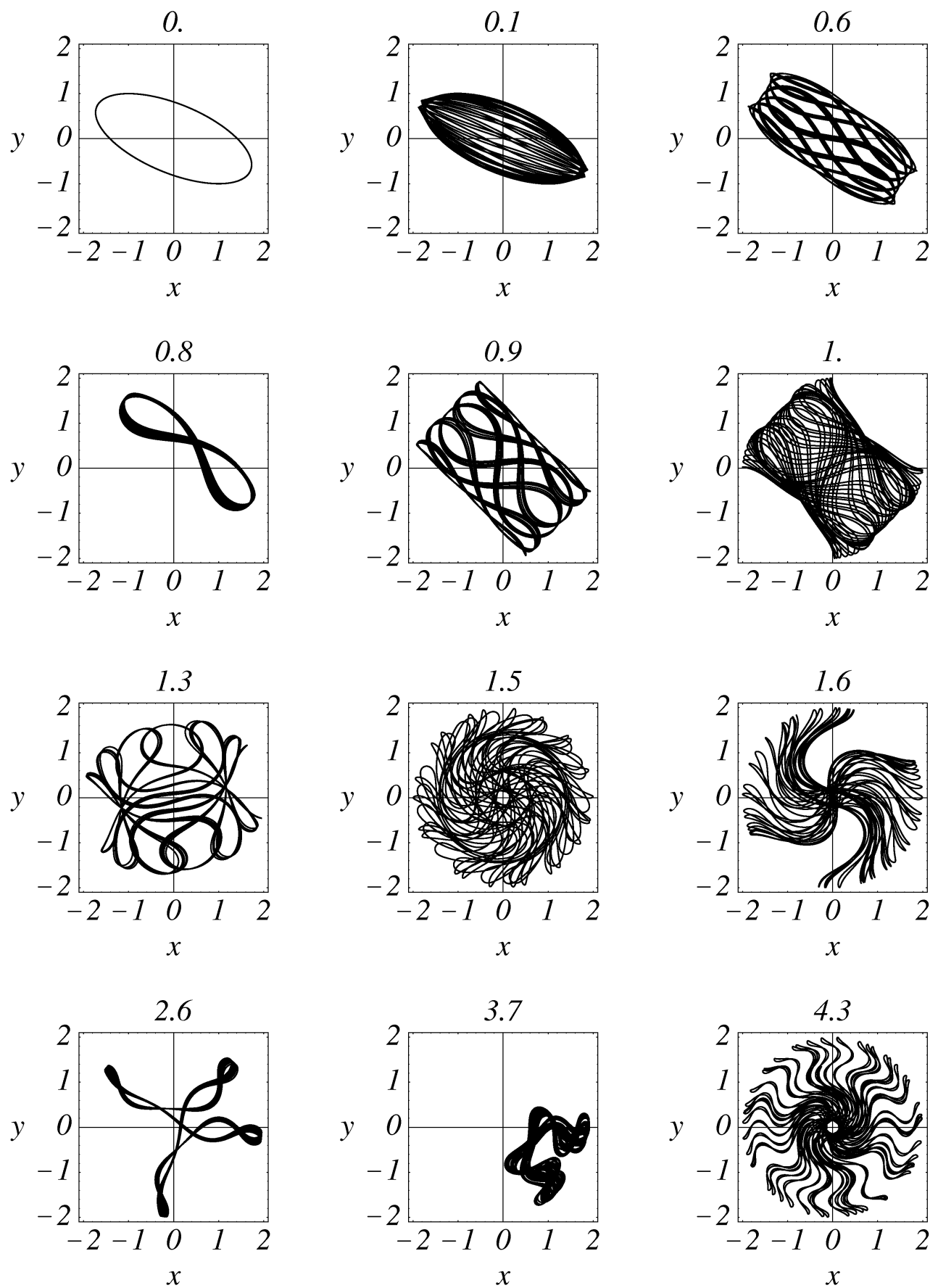

Fig. 14. The orbits of the adiabatic harmonic oscillator subjected to twirlers with selected stirring coupling coefficients $\zeta_{\mathrm{t}}$ ranging from 0.0 to 4.3 . The orbits are shown over $64 \pi \approx 200$ time units.

$0.0, v_{x}=\sqrt{0.15}$, and $v_{y}=-\sqrt{0.15}$, which imply energy $E=0.15$, are presented in Fig. 9 and in the inital kinetic energy $E_{\text {kin }}=0.15$ and the total Table 4 . 
[1] W. G. Hoover Molecular Dynamics, Springer, Berlin 1986; Computational Statistical Mechanics, Elsevier, Amsterdam 1991.

[2] M. P. Allen and D. J. Tildesley, Computer Simulation of Liquids, Clarendon, Oxford 1987.

[3] D. J. Evans and G. P. Morriss, Statistical Mechanics of Nonequilibrium Liquids, Academic Press, London 1990.

[4] S. Nose, J. Chem. Phys. 81, 551 (1984); W. G. Hoover, Phys. Rev. A 31, 4757 (1985).

[5] W. G. Hoover, Phys. Rev. A 40, 2814 (1989); B.L. Holian, W. G. Hoover, and H. Posch, Phys. Rev. Lett. 59, 10 (1987); K. Rateitschak, R. Klages, and W. G. Hoover, J. Stat. Phys. 101, 61 (2000).

[6] A. Bulgac and D. Kusnezov, Phys. Rev. A 42, 5045 (1990).

[7] R. Klages, K. Rateitschak, and G. Nicolis, Phys. Rev. Lett. 84, 4268 (2000); C. Wagner, R. Klages, and G. Nicolis, Phys. Rev. E 60, 1401 (1999); K. Rateitschak and R. Klages, Phys. Rev. E 65, 036209-1-11 (2002).

[8] H. H. Rugh, Phys. Rev. Lett. 78, 772 (1997); O. G. Jeps, G. Ayton, and D. J. Evans, Phys. Rev. B 62, 4757 (2000).

[9] S. Hess, M. Kröger, W. Loose, C. Pereira Borgmeyer, R. Schramek, H. Voigt, and T. Weider, Simple and
Complex Fluids under Shear, in K. Binder and G. Ciccotti (eds.) Monte Carlo and Molecular Dynamics of Condensed Matter Systems, IPS Conf. Proc. 49, Bologna 1996, p. 825-841.

[10] S. Hess and G. P. Morriss, to be published.

[11] C. Aust, S. Hess, and M. Kröger, Macromolecules 35, 8621 (2002).

[12] S. Hess and L. Waldmann, Z. Naturforsch. 21a, 1529 (1966); 25a, 1367 (1970).

[13] A. G. St. Pierre, W. E. Köhler, and S. Hess, Z. Naturforsch. 27a, 721 (1972).

[14] S. Hess, Phys. Lett. 30A, 239 (1969).

[15] T. Weider, U. Stottut, W. Loose, and S. Hess, Physica A 174, 1 (1991).

[16] S. Hess, Z. Naturforsch. 23a, 597 (1968); Physica 74, 277 (1974).

[17] S. Hess and D. Isbister, preliminary results.

[18] R. Cerf, J. Chim. Phys. 68, 479 (1969); E. R. Bazua and M. C. Williams, J. Polym. Sci. 12, 825 (1974).

[19] Th. Dorfmüller and R. Pecora, Eds., Rotational dynamics of small and Macromolecules, Lecture Notes in Physics 293, Springer, Berlin 1987.

[20] E. Gedat, A. Schreiber, G. Findenegg, H. H. Limbach, and G. Buntkowsky, J. Phys. Chem. B 106, 1977 (2003). 